\title{
On the microscopic nature of inhomogeneously broadened spectra of chromophores in glasses and crystals
}

\author{
Brian B. Laird and J. L. Skinner \\ Department of Chemistry, Columbia University, New York, New York 10027
}

(Received 12 December 1988; accepted 24 January 1989)

Inhomogeneous broadening in the optical spectra of impurity chromophores in crystals and glasses is a manifestation of a static distribution of impurity environments, which gives rise to a distribution of the absolute energies for a given impurity electronic state. The inhomogeneous line shape for a transition from state $\alpha$ to state $\beta$ is obtained from a convolution of the probability distribution for the energy of state $\alpha$, $p_{\alpha}(E)$, and $f_{\beta \alpha}\left(E^{\prime} \mid E\right)$, the conditional probability that a chromophore has energy $E^{\prime}$ in state $\beta$ given that it had energy $E$ in state $\alpha$. Experiments that involve a transition between two states followed by radiative or nonradiative relaxation to a third state, such as fluorescence line narrowing (FLN) or hole burning (HB), provide additional interesting information about these absolute distributions and conditional probabilities.

In a 1985 paper, ${ }^{1}$ Lee, Walsh, and Fayer (LWF) proposed a phenomenological model of inhomogeneous broadening, which they used to explain a series of FLN and HB experiments. They assumed that the energy distributions of the individual states are Gaussian in form, and that the conditional probabilities $f_{\beta \alpha}\left(E^{\prime} \mid E\right)$ are simply equal to $p_{\beta}\left(E^{\prime}\right)$. Thus all the pairs of absolute energies are completely uncorrelated. The model of LWF has recently been generalized by Suter et al. ${ }^{2}$ In an alternative viewpoint, which has been discussed by Selzer, ${ }^{3}$ one assumes that there is a one-to-one correspondence between the energy in one state, and the energy in some other state, so that the conditional probabilities would all be delta functions. Inhomogeneous broadening arises in this model because the absolute energy distributions for the two levels have different widths.

In a recent paper, ${ }^{4}$ we have developed a molecular theory that describes the response of the inhomogeneous line shape after a persistent hole has been burned, to external changes in pressure. This effort was motivated by experiments performed by Sesselmann et al. ${ }^{5}$ We came to realize that, using a modified version of our formalism, it is possible to develop a theory within which the energy distributions and conditional probabilities discussed phenomenologically by LWF and Selzer can be calculated from the microscopic parameters of a system. In what follows, we will briefly outline this theory and comment on how the LWF and Selzer assumptions are recovered in certain limits.

We consider a system consisting of a dilute concentration of impurities embedded in a glass or crystal. In some zeroth-order scheme, the impurities are characterized by a set of levels $\{\alpha\}$ with energies $E_{\alpha}^{0}$. We then suppose that each impurity interacts with a large number of static "defects" (which for a crystal would include dislocations, interstitials, etc., and for a glass would be the solvent molecules themselves ${ }^{4}$ ), each of which produces a perturbation $v_{\alpha}(\mathbf{R})$ on the $\alpha$ th impurity level, where $\mathbf{R}$ is the set of relevant defect coordinates, including its position relative to the impurity. The (normalized) distribution of state $\alpha$ energies is then given by

$$
\begin{aligned}
p_{\alpha}(E)= & V^{-N} \int d \mathbf{R}_{1} \ldots d \mathbf{R}_{N} P\left(\mathbf{R}_{1}, \ldots, \mathbf{R}_{N}\right) \\
& \times \delta\left(E-\left[E_{\alpha}^{0}+\sum_{i=1}^{N} v_{\alpha}\left(\mathbf{R}_{i}\right)\right]\right),
\end{aligned}
$$

where $P\left(\mathbf{R}_{1}, \ldots, \mathbf{R}_{N}\right)$ is the probability distribution function for the coordinates of the $N$ defects, normalized to $V^{N}$, where $V$ is the volume of the system. Following the procedure outlined in Ref. 4, i.e., making the approximation that these defect coordinates are uncorrelated so that $P\left(\mathbf{R}_{1}, \ldots, \mathbf{R}_{N}\right)$ can be written as a product of single defect distribution functions $g\left(\mathbf{R}_{i}\right)$, we find that for a sufficiently large defect density $\rho=N / V$, the function $p_{\alpha}(E)$ is a normalized Gaussian centered at

$$
E_{\alpha}=E_{\alpha}^{0}+\rho \int d \mathbf{R} g(\mathbf{R}) v_{\alpha}(\mathbf{R}),
$$

with standard deviation $\left(D_{\alpha \alpha}\right)^{1 / 2}$, where

$$
D_{\alpha \beta} \equiv \rho \int d \mathbf{R} g(\mathbf{R}) v_{\alpha}(\mathbf{R}) v_{\beta}(\mathbf{R})
$$

Within the same model, the conditional probability connecting two states $\alpha$ and $\beta$ is given by

$$
\begin{aligned}
f_{\beta \alpha}\left(E^{\prime} \mid E\right)= & \frac{1}{p_{\alpha}(E) V^{N}} \int d \mathbf{R}_{1} \ldots d \mathbf{R}_{N} P\left(\mathbf{R}_{1}, \ldots, \mathbf{R}_{N}\right) \\
& \times \delta\left(E-\left[E_{\alpha}^{0}+\sum_{i=1}^{N} v_{\alpha}\left(\mathbf{R}_{i}\right)\right]\right) \\
& \times \delta\left(E^{\prime}-\left[E_{\beta}^{0}+\sum_{i=1}^{N} v_{\beta}\left(\mathbf{R}_{i}\right)\right]\right)
\end{aligned}
$$

The same set of approximations that lead to the Gaussian form for the energy distributions also yield a normalized Gaussian (in $E^{\prime}$ ) for the conditional probability, centered at $E_{\beta}+\left(E-E_{\alpha}\right)\left(D_{\alpha \beta} / D_{\alpha \alpha}\right)$, and with standard deviation $\left[D_{\beta \beta}-\left(D_{\alpha \beta}^{2} / D_{\alpha \alpha}\right)\right]^{1 / 2}$.

There are two important limits to consider. First, if the potential $v_{\beta}(\mathbf{R})=\lambda v_{\alpha}(\mathbf{R})$, where $\lambda$ is a constant, then the conditional probability $f_{\beta \alpha}\left(E^{\prime} \mid E\right)$ is a delta function: $\delta\left(E^{\prime}-E_{\beta}-\left(E-E_{\alpha}\right) \lambda\right)$. Thus the two states are completely correlated, which is the Selzer limit. As an example of this case, consider an impurity with a permanent dipole moment in state $\alpha$ interacting with randomly distributed dipolar defects. If in state $\beta$ the permanent dipole changes magnitude but not direction, then $v_{\beta}(\mathbf{R})=\lambda v_{\alpha}(\mathbf{R})$. Another example involves an impurity that interacts via a LennardJones potential with randomly positioned solvent molecules. 
In the approximation that only the $\epsilon$ (and not the $\sigma$ ) parameter of the Lennard-Jones interaction changes in going from state $\alpha$ to state $\beta$, this again gives $v_{\beta}(\mathbf{R})=\lambda v_{\alpha}(\mathbf{R})$.

However, for other models of the impurity-defect interaction where $v_{\beta}(\mathbf{R}) \neq \lambda v_{\alpha}(\mathbf{R})$, the conditional probability will be Gaussian with a width given above. For potentials such that $D_{\alpha \beta} \ll D_{\alpha \alpha}, D_{\beta \beta}$ [see Eq. (3)], then $f_{\beta \alpha}\left(E^{\prime} \mid E\right) \approx p_{\beta}\left(E^{\prime}\right)$, which is the uncorrelated LWF ansatz. LWF argue that a wide variety of experiments, especially an interesting antihole separation study, ${ }^{1}$ can be understood on the basis of the uncorrelated model. However, this conclusion rests on making additional assumptions involving the correlations between the states of the original chromophore and the photoproduct. Within the microscopic framework described above, the correlations between all states arise naturally, and hopefully it will be possible to analyze these experiments in detail.

Once the energy distributions and conditional probabilities are determined, any experimental observable can then be calculated. For example, the $\alpha \rightarrow \beta$ transition line shape can be obtained by

$I_{\beta \alpha}(\Delta E)$

$$
=\int d E \int d E^{\prime} \delta\left(E^{\prime}-E-\Delta E\right) f_{\beta \alpha}\left(E^{\prime} \mid E\right) p_{\alpha}(E)
$$

Evaluating Eq. (5) gives a Gaussian line shape (in $\Delta E$ ) centered at $E_{\beta}-E_{\alpha}$, and with standard deviation $\left(D_{\beta \beta}+D_{\alpha \alpha}-2 D_{\alpha \beta}\right)^{1 / 2}$. As a final example, consider a FLN experiment where the $\alpha \rightarrow \beta$ transition is excited with a narrow band laser, and the line shape of the ensuing fluorescence from the $\beta$ state to a third state $\gamma$ is studied. The fluorescence line shape, as a function of the initial laser energy $E_{L}$, can be written in terms of the initial $\alpha$ state distribution and the conditional probabilities connecting the $\alpha$ and $\beta$ states and the $\beta$ and $\gamma$ states, as

$$
\begin{aligned}
I_{\gamma \beta}\left(\Delta E ; E_{L}\right)= & \int d E \int d E^{\prime} \int d E^{\prime \prime} \delta\left(E^{\prime}-E^{\prime \prime}-\Delta E\right) \\
& \times \delta\left(E^{\prime}-E-E_{L}\right) f_{\gamma \beta}\left(E^{\prime \prime} \mid E^{\prime}\right) \\
& \times f_{\beta \alpha}\left(E^{\prime} \mid E\right) p_{\alpha}(E) .
\end{aligned}
$$

It is then straightforward to evaluate Eq. (6) in terms of the microscopic parameters.

To conclude, this communication shows how the conditional probabilities and absolute energy distributions needed to interpret a variety of optical experiments can be obtained from a microscopic model of inhomogeneous broadening. Specific choices for the perturbations $v_{\alpha}(\mathbf{R})$ can then be invoked to analyze results from different experimental systems.

This research is supported by the National Science Foundation under Grant Nos. DMR86-03394 and CHE8351207, and by the Alfred P. Sloan and Camille and Henry Dreyfus Foundations.

'H. W. H. Lee, C. A. Walsh, and M. D. Fayer, J. Chem. Phys. 82, 3948 (1985).

${ }^{2}$ G. W. Suter, U. P. Wild, and A. R. Holzwarth, Chem. Phys. 102, 205 (1986).

${ }^{3}$ P. M. Selzer, in Laser Spectroscopy of Solids, edited by W. M. Yen and P. M. Selzer (Springer, Berlin, 1981), p. 113.

${ }^{4}$ B. B. Laird and J. L. Skinner, J. Chem. Phys. 90, 3274 (1989).

${ }^{5}$ Th. Sesselmann, W. Richter, D. Haarer, and H. Morawitz, Phys. Rev. B 36, 7601 (1987).

\title{
Alignment of $\mathbf{I}_{\mathbf{2}}$ molecules seeded in a supersonic beam
}

\author{
D. P. Pullman and D. R. Herschbach \\ Department of Chemistry, Harvard University, Cambridge, Massachusetts 02138
}

(Received 21 December 1988; accepted 25 January 1988)

Fifty years ago Gorter pointed out a collisional mechanism for alignment of the rotational angular momentum vector $\mathbf{j}$ of a diatomic molecule. ${ }^{1}$ If the relative velocity vectors in the collisions have a preferred spatial direction $\mathbf{k}$, molecules rotating with $\mathbf{j}$ parallel to $\mathbf{k}$ present a broadside target and thus suffer drastic randomizing impacts more often than those with $\mathbf{j}$ perpendicular to $\mathbf{k}$. The alignment produced may be described by a Legendre expansion, $1+a_{2} P_{2}(\hat{\mathbf{j}} \cdot \hat{\mathbf{k}})+\cdots$. Only even order terms appear and $a_{2}$ is negative when $\mathbf{j}$ prefers to be perpendicular to $\mathbf{k}$. Gorter's alignment mechanism has been invoked in models for polarization of interstellar absorption lines, ${ }^{2}$ transport properties of gases, ${ }^{3}$ and many other phenomena. It has also long been recognized $^{4}$ that this mechanism might be exploited in supersonic beams to enable studies of steric effects in collision processes, especially the stereodynamics of chemical reactions. $^{5}$

However, as yet the only experimental demonstrations of substantial rotational alignment in supersonic beams pertain to alkali dimers ${ }^{6-9}$ (with $a_{2}$ as large as -0.4 ). Similar experiments have found puny alignment for neat iodine beams ${ }^{7}\left(a_{2}\right.$ only -0.0056$)$ and equivocal results for iodine seeded in a $\mathrm{CO}_{2}$ beam. ${ }^{4}$ This accounts for the prevalent view, which regards the alkali dimers as exceptional (due to unusually long bond lengths or facile atom exchange) and assumes rotational alignment in supersonic expansions is typi- 